\title{
ON LIPSCHITZ BALL NONCOLLAPSING FUNCTIONS AND UNIFORM CO-LIPSCHITZ MAPPINGS OF THE PLANE
}

OLGA MALEVA

Received 29 October 2003

We give a sharp estimate on the cardinality of point preimages of a uniform co-Lipschitz mapping on the plane. We also give a necessary and sufficient condition for a ball noncollapsing Lipschitz function to have a point with infinite preimage.

\section{Introduction}

Consider a mapping $f: X \rightarrow Y$ between two normed spaces $X$ and $Y$. The function

$$
\Omega_{f}(d)=\sup _{\left\|x-x^{\prime}\right\|_{X} \leq d}\left\|f(x)-f\left(x^{\prime}\right)\right\|_{Y}
$$

is called the modulus of (uniform) continuity of $f$. The mapping $f$ is said to be uniformly continuous if $\Omega_{f}(d) \rightarrow 0$ as $d \downarrow 0$. In this case the modulus of continuity is a subadditive monotone continuous function. The definition of $\Omega_{f}$ implies that $f\left(\bar{B}_{r}(x)\right) \subset$ $\bar{B}_{\Omega_{f}(r)}(f(x))$. (By $B_{\rho}(y)$ and $\bar{B}_{\rho}(y)$ we denote, respectively, the open and the closed ball of radius $\rho$, centered at $y$.)

One important class of uniformly continuous mappings is the class of Lipschitz mappings, that is, those satisfying $\Omega_{f}(d) \leq L d$ for some positive $L$. The least such $L$ is called the Lipschitz constant of the mapping $f$.

In a similar way, couniformly continuous mappings are defined as those satisfying

$$
f\left(B_{r}(x)\right) \supset B_{\omega(r)}(f(x)), \quad r>0,
$$

where $\omega(r)$ is a function of the radius $r$ independent of the point $x$, such that $\omega(r)>0$ for $r>0$. A particular case is a co-Lipschitz mapping which satisfies

$$
f\left(B_{r}(x)\right) \supset B_{c r}(f(x)) .
$$

We call the best (the largest) such constant $c$ the co-Lipschitz constant of the mapping $f$. (Note that in some papers, in particular [3], the co-Lipschitz constant is the reciprocal of our c.)

A mapping $f$ is called a uniform quotient if it is both uniform and couniform; $f$ is called a Lipschitz quotient if it is Lipschitz and co-Lipschitz. 
In Section 2, we generalize results which were obtained in $[4,5]$. We show that for a uniform co-Lipschitz mapping of the plane, the cardinality of the preimage of a point may be estimated in terms of the characteristic constants of the mapping, that is, its co-Lipschitz and weak Lipschitz constants, namely, the maximum number of points that a mapping $f$ can glue together does not exceed $L_{f}^{*} / c$. The weak Lipschitz constant $L_{f}^{*}$ of a uniform mapping $f$ is defined in the following way:

$$
L_{f}^{*}=\lim _{d \rightarrow+\infty} \frac{\Omega_{f}(d)}{d}<+\infty
$$

(see [6] for a discussion of this constant). If $f$ is a Lipschitz mapping, $L_{f}^{*}$ does not exceed its Lipschitz constant (it can, however, be strictly less than the Lipschitz constant). If the mapping is uniform with weak Lipschitz constant $L_{f}^{*}$ and is $c$-co-Lipschitz, then $c \leq L_{f}^{*}$.

In this paper, we will use the notion of the index (also called winding number) of a closed curve around a point not on the curve, and the notion of $n$-dimensional Hausdorff measure:

$$
\mathscr{H}_{n}(A)=\sup _{\delta>0} \inf \left\{\sum_{j=1}^{\infty}\left(\frac{\operatorname{diam} C_{j}}{2}\right)^{n} \mid A \subset \bigcup_{j=1}^{\infty} C_{j}, \operatorname{diam} C_{j} \leq \delta\right\}
$$

(cf. $[1,2.8 .15])$. Of course, the diameter in this definition is with respect to the metric given by the norm. Note that $\mathscr{H}_{n}$ is so normalized that the measure of the unit ball is equal to 1 .

We also settle a special case of the volume ratio problem: if $f$ is a Lipschitz quotient mapping of the plane, then for any measurable set $A, \lambda_{2}(f(A)) / \lambda_{2}(A)$ is bounded from below by a positive constant depending only on the Lipschitz and co-Lipschitz constants of $f$.

In Section 3, we deal with so-called ball noncollapsing mappings (see $[2,5]$ ).

A mapping $f$ is called ball noncollapsing, if the $f$-image of a ball of radius $r$ always contains a ball of radius $C r$, where $C$ is a positive constant. The largest such $C$ is called the $\mathrm{BNC}$ constant of the mapping. The difference between BNC and co-Lipschitz mappings is that the ball of radius $\mathrm{Cr}$ contained in $f\left(B_{r}(x)\right)$ need not be centered at $f(x)$; the class of BNC mappings is actually strictly wider than that of co-Lipschitz mappings.

In [5], we proved that if $f: \mathbb{R}^{2} \rightarrow \mathbb{R}^{2}$ is $L$-Lipschitz and $C$-BNC with $C / L>1 / 2$, then $f$ is one-to-one. The same statement can be easily shown to be true for $f: \mathbb{R}^{1} \rightarrow \mathbb{R}^{1}$ (such a mapping has to be co-Lipschitz by [5, Lemma 4], and therefore, is monotone).

However, when $C / L \leq 1 / 2$, the mapping is not necessarily one-to-one (consider $f(x)=$ $|x|$ on $\left.\mathbb{R}^{1}\right)$. In the present paper, we prove that for any pair of positive constants $(C, L)$ the following are equivalent:

(i) there exists $f: \mathbb{R}^{1} \rightarrow \mathbb{R}^{1}, C$-BNC and L-Lipschitz, and a point $x \in \mathbb{R}^{1}$ such that $f^{-1}(x)$ is infinite;

(ii) $C / L<1 / 3$. 


\section{Uniform co-Lipschitz mappings of the plane}

In the first part of the paper, we show that under a uniform co-Lipschitz mapping, a point may have up to $L_{f}^{*} / c$ preimages. Since for a Lipschitz mapping its weak Lipschitz constant does not exceed its Lipschitz constant, we see that Theorem 2.1 generalizes our previous result presented in [5, Theorem 1] (which holds for Lipschitz mappings with $L$ instead of $\left.L_{f}^{*}\right)$.

Theorem 2.1. Let $\|\cdot\|$ be any norm on $\mathbb{R}^{2}$. If $f:\left(\mathbb{R}^{2},\|\cdot\|\right) \rightarrow\left(\mathbb{R}^{2},\|\cdot\|\right)$ is uniform co-Lipschitz, with c being its co-Lipschitz constant and $L_{f}^{*}$ its weak Lipschitz constant, and

$$
\max _{x \in \mathbb{R}^{2}} \# f^{-1}(x)=n
$$

then $c / L_{f}^{*} \leq 1 / n$.

This theorem immediately yields the existence of the following scale.

COROllary 2.2. There is a scale of numbers

$$
0<\cdots<C_{2}^{(n)}<\cdots<C_{2}^{(1)}=C_{2}<1
$$

with $C_{2}^{(n)}=1 /(n+1)$ such that for any norm $\|\cdot\|$ of the plane, and any uniform co-Lipschitz mapping $f:\left(\mathbb{R}^{2},\|\cdot\|\right) \rightarrow\left(\mathbb{R}^{2},\|\cdot\|\right)$, the condition $c / L_{f}^{*}>C_{2}^{(n)}$ implies $\# f^{-1}(x) \leq n$ for any $x \in \mathbb{R}^{2}$.

Remark 2.3. Once we have such a scale, a natural question is whether the $1 / n$ bounds are precise. In the case of $\|\cdot\|$ being the Euclidean norm, the "winding mapping" $\phi_{n}\left(r e^{i \theta}\right)=$ $r e^{n i \theta}$ has weak Lipschitz constant $n$ and co-Lipschitz constant 1 , so the ratio of constants $L_{\phi_{n}}^{*} / c$ is equal to the maximum cardinality of a point preimage, which is $n$.

An analogue of the winding mapping can be constructed for arbitrary norm $\|\cdot\|$. One can define the argument, $\arg _{\|\cdot\|}(y)$, of any nonzero point $y$, and then set $\psi_{n}(r x)=r y$, where $r \geq 0$, and $y$ is a point on $\partial B_{1}$ such that $\arg _{\|\cdot\|}(y)=n \arg _{\|\cdot\|}(x)$ (see $[5$, Section 3] for the description of this construction). In the situation when the unit ball is a regular polygon (or, of course, its affine equivalent), the weak Lipschitz constant of $\psi_{n}$ is then shown to be equal to $n$, the co-Lipschitz constant of $\psi_{n}$ is 1 , so again $L_{\psi_{n}}^{*} / c=n$.

We have not yet worked out this example for other norms, so despite the feeling that the estimate is sharp for any given norm (i.e., there exists a mapping $f$ with a maximum of $n$ point preimages and the ratio of constants $L_{f}^{*} / c$ equal to $n$ ), this question remains open.

Proof of Theorem 2.1. Without loss of generality we may assume that $f(0)=0$. By [3] there exist a homeomorphism $h: \mathbb{R}^{2} \rightarrow \mathbb{R}^{2}$ and a polynomial $P(z)$ of one complex variable, such that

$$
f=P \circ h
$$

Clearly, $\operatorname{deg} P=\max _{x \in \mathbb{R}^{2}} \# f^{-1}(x)=n$. If $n=1$, then the statement is obvious. Assume $n \geq 2$. 
Assume that $c / L_{f}^{*}>1 / n$, then by rescaling without loss of generality we may assume that $L_{f}^{*}<1$ and $c>1 / n$. Fix any

$$
c_{1} \in\left(\frac{1}{n}, c\right)
$$

Let

$$
\varepsilon=\frac{c_{1}-c}{c}
$$

then $c_{1}=c(1-\varepsilon)$.

Changing $h$ by a transformation of the form $h \rightarrow a h+b$, we may assume that $h(0)=0$ and the leading coefficient $a_{n}$ of $P(z)$ is 1 . Then $P(0)=f(0)=0$ and $P(z)$ has the form $z^{n}+a_{n-1} z^{n-1}+\cdots+a_{1} z$.

We consider $\mathbb{R}^{2}$ as the complex plane, and use the notation $|x|$ for the absolute value of the complex number $x$, which is the same as the Euclidean norm of $x \in \mathbb{R}^{2}$.

Let $\left\{z_{1}=0, z_{2}, \ldots, z_{k}\right\}$ be the set of preimages of zero under $f$, denote

$$
M=\max _{1 \leq i \leq k}\left\|z_{i}\right\|
$$

Lemma 2.4. If $f$ is such as described in the hypothesis of Theorem 2.1, and $c_{1}$ is as in (2.4), then there exists an $R$ such that for any $x$ with $\|x\| \geq R,\|f(x)\| \geq c_{1}\|x\|$.

Before stating the next lemma, we recall the notation $\operatorname{Ind}_{0} \gamma$ for the index around the origin of a closed curve $\gamma$.

Lemma 2.5. There exists $d>1$ such that for any $\rho>d$,

$$
\operatorname{Ind}_{0} f\left(\partial B_{\rho}(0)\right)=\operatorname{Ind}_{0} P\left(h\left(\partial B_{\rho}(0)\right)\right)=n .
$$

Lemma 2.6. If $\Gamma:[0,1] \rightarrow \mathbb{R}^{2}$ is a closed curve with $\|\Gamma(t)\| \geq r$ for all $t \in[0,1]$ and $\operatorname{Ind}_{0} \Gamma=$ $n$, then the length of $\Gamma$ in the sense of the 1-dimensional Hausdorff measure $\mathscr{H}_{1}$ is at least

$$
n \mathscr{H}_{1}\left(\partial B_{r}(0)\right) \text {. }
$$

For the proof of Lemma 2.4 see [5, Lemma 1] and [4, Lemma 1], for the proof of Lemma 2.5 see [5, Lemma 2], and for the proof of Lemma 2.6 see [5, Lemma 3].

Now we return to the proof of Theorem 2.1.

Consider $S=\partial B_{1}$ - the unit sphere in the norm $\|\cdot\|$, as a closed, central symmetric curve in $\left(\mathbb{R}^{2},|\cdot|\right)$. For each $\varepsilon$ we denote by $P(\varepsilon)$ a polygon inscribed in $S$ with the following property: the Euclidean length of each arc between two adjacent vertices of $P(\varepsilon)$ is less than $\varepsilon$.

We assumed in the beginning of the proof that $L_{f}^{*}<1$, therefore there exists $d_{0}$ such that $\Omega_{f}(d)<d$ for all $d \geq d_{0}$.

Let $a$ be the constant of equivalence between the norm $\|\cdot\|$ and the Euclidean norm $|\cdot|$, that is,

$$
a^{-1}|x| \leq\|x\| \leq a|x| \quad \forall x
$$


Let $\varepsilon_{0}=\min \left\{c_{1} / 2 a, d_{0} / a\right\}$ and assume $\varepsilon<\varepsilon_{0}$. Note that $\|\cdot\|$-lengths of the sides of the polygon $P(\varepsilon)$ are less than or equal to $a \varepsilon$, which is less than $d_{0}$. Let $\ell(\varepsilon)$ be the smallest $\|\cdot\|$-length among the lengths of sides of $P(\varepsilon)$.

Consider the rescaling of $P(\varepsilon)$ by a factor of $d_{0} / \ell(\varepsilon)$, and denote the new polygon by $A_{1} A_{2} \cdots A_{m}$ (of course, the vertices $A_{i}$ 's and their total number $m$ depend on $\varepsilon$ ). For each $i$ one has $\left\|A_{i}-A_{i+1}\right\| \geq d_{0}$, hence $\left\|f A_{i}-f A_{i+1}\right\| \leq \Omega_{f}\left(\left\|A_{i}-A_{i+1}\right\|\right)<\left\|A_{i}-A_{i+1}\right\|$. Thus the $\mathscr{H}_{1}$-length $\mathscr{L}$ of the broken line $f\left(A_{1}\right) f\left(A_{2}\right) \cdots f\left(A_{m}\right)$ does not exceed the $\mathscr{H}_{1}$-length of the broken line $A_{1} A_{2} \cdots A_{m}$, which is not more than $\mathscr{H}_{1}\left(\partial B_{d_{0} / \ell(\varepsilon)}\right)=\left(d_{0} / \ell(\varepsilon)\right) \mathscr{H}_{1}\left(\partial B_{1}\right)$.

Now we are going to estimate $\mathscr{L}$ from below. For this purpose, we first prove that Ind $_{0} f A_{1} f A_{2} \cdots f A_{m}=n$ for sufficiently small $\varepsilon$.

By Lemmas 2.4 and 2.5 , there exists $R$ such that $\|f(x)\|>c_{1}\|x\|$ for all $x$ such that $\|x\| \geq R$, and $\operatorname{Ind}_{0} f\left(\partial B_{r}\right)=n$ for all $r \geq R$.

Since $\ell(\varepsilon) \leq a \varepsilon$ (all sides of the polygon $P(\varepsilon)$ were of $\|\cdot\|$-length less than or equal to $a \varepsilon)$, one has $\ell(\varepsilon) \rightarrow 0$ as $\varepsilon \rightarrow 0$, and so $d_{0} / \ell(\varepsilon) \rightarrow+\infty$ as $\varepsilon \rightarrow 0$. Take such $\varepsilon_{1}<\varepsilon_{0}$ so that $d_{0} / \ell(\varepsilon)>R$ for any positive $\varepsilon<\varepsilon_{1}$ and fix some $\varepsilon \in\left(0, \varepsilon_{1}\right)$.

Note that the $\|\cdot\|$-distance between $A_{i}$ and any point on the arc $\mathscr{A}_{i, i+1}=A_{i} A_{i+1}$ of the $\|\cdot\|$-sphere of radius $d_{0} / \ell(\varepsilon)$ centered at zero is less than or equal to $a \varepsilon \cdot d_{0} / \ell(\varepsilon)$ (this can be easily demonstrated using triangle inequality), so $\mathscr{A}_{i, i+1} \subset \bar{B}_{a \varepsilon d_{0} / \ell(\varepsilon)}\left(A_{i}\right)$. Thus

$$
f\left(\mathscr{A}_{i, i+1}\right) \subset \bar{B}_{\Omega_{f}\left(a \varepsilon d_{0} / \ell(\varepsilon)\right)}\left(f A_{i}\right)
$$

Since $a \varepsilon d_{0} / \ell(\varepsilon) \geq d_{0}$, the choice of $d_{0}$ yields

$$
\Omega_{f}\left(\frac{a \varepsilon d_{0}}{\ell(\varepsilon)}\right)<\frac{a \varepsilon d_{0}}{\ell(\varepsilon)},
$$

and therefore

$$
\bar{B}_{\Omega_{f}\left(a \varepsilon d_{0} / \ell(\varepsilon)\right)}\left(f A_{i}\right) \subset B_{a \varepsilon d_{0} / \ell(\varepsilon)}\left(f A_{i}\right)
$$

Denote the latter ball by $B$. Note that by the choice of $\varepsilon_{0}$, the radius of $B$ is less than $\left(c_{1} / 2\right) d_{0} / \ell(\varepsilon)$, and at the same time $\left\|f A_{i}\right\|>c_{1}\left\|A_{i}\right\|=c_{1} d_{0} / \ell(\varepsilon)$, so $B \not 0$. Therefore,

$$
n=\operatorname{Ind}_{0} f \partial B_{d_{0} / \ell(\varepsilon)}=\operatorname{Ind}_{0} f A_{1} f A_{2} \cdots f A_{m}
$$

since if we replace $f\left(\mathscr{A}_{i, i+1}\right)$ by the segment $\left[f A_{i}, f A_{i+1}\right]$, the total index does not change.

Now we estimate the $\|\cdot\|$-distance of each segment $I_{i}=\left[f A_{i}, f A_{i+1}\right]$ to zero. The length of $I_{i}$, as we already showed is less than $\left\|A_{i}-A_{i+1}\right\| \leq a \varepsilon d_{0} / \ell(\varepsilon)$, and both its ends have norm at least $c_{1} d_{0} / \ell(\varepsilon)$. Thus $\operatorname{dist}\left(I_{i}, 0\right) \geq\left(1-a \varepsilon / c_{1}\right) c_{1} d_{0} / \ell(\varepsilon)$.

Hence by Lemma 2.6, $\mathscr{L} \geq n \cdot\left(1-a \varepsilon / c_{1}\right) c_{1}\left(d_{0} / \ell(\varepsilon)\right) \mathscr{H}_{1}\left(\partial B_{1}\right)$. Thus, we get the following inequality:

$$
\frac{d_{0}}{\ell(\varepsilon)} \mathscr{H}_{1}\left(\partial B_{1}\right) \geq n \cdot\left(1-\frac{a \varepsilon}{c_{1}}\right) c_{1} \frac{d_{0}}{\ell(\varepsilon)} \mathscr{H}_{1}\left(\partial B_{1}\right)
$$


or, equivalently,

$$
1 \geq n c_{1}\left(1-\frac{a \varepsilon}{c_{1}}\right)
$$

which does not hold for sufficiently small $\varepsilon$, since the right-hand side tends to $n c_{1}>1$ as $\varepsilon \rightarrow 0$.

We arrived at this contradiction because we assumed in the beginning that $c>1 / n$, which enabled us to choose $c>c_{1}>1 / n$. Thus, $c \leq 1 / n$, and the theorem is proved.

As a corollary, we obtain an interesting result: a Lipschitz quotient mapping does not collapse areas.

Theorem 2.7. If $f: \mathbb{R}^{2} \rightarrow \mathbb{R}^{2}$ is an L-Lipschitz and c-co-Lipschitz mapping with respect to the Euclidean norm, then for any measurable set $A \subset \mathbb{R}^{2}$ of finite positive 2-dimensional Lebesgue measure $\lambda_{2}(A)$,

$$
\frac{c^{3}}{L} \leq \frac{\lambda_{2}(f(A))}{\lambda_{2}(A)} .
$$

Proof. Let $J f$ be the Jacobian of $f$ (which is defined almost everywhere on $\mathbb{R}^{2}$ since $f$ is Lipschitz). By the coarea formula (see $[1,3.211]$ ),

$$
\int_{A}|J f| d x=\int_{\mathbb{R}^{2}} \#\left(A \cap f^{-1}(y)\right) d y .
$$

Let $N$ be the maximum cardinality of a point preimage under $f$. By Theorem 2.1, $N$ does not exceed $L / c$. Since $\#\left(A \cap f^{-1}(y)\right) \neq 0$ is equivalent to $y \in f(A)$, we conclude

$$
\int_{\mathbb{R}^{2}} \#\left(A \cap f^{-1}(y)\right) d y \leq N \lambda_{2}(f(A)) \leq\left(\frac{L}{c}\right) \lambda_{2}(f(A)) .
$$

But obviously $|J f| \geq c^{2}$ almost everywhere, thus the left-hand side of (2.17) is at least $c^{2} \lambda_{2}(A)$. Thus, $\lambda_{2}(f(A)) \geq\left(c^{3} / L\right) \lambda_{2}(A)$.

Note that if $f$ is a Lipschitz quotient (Lipschitz and co-Lipschitz) and (2.16) holds for every measurable set $A$, then the preimage of each point is finite. In fact, any estimate

$$
\lambda_{2}(f(A)) \geq \delta \lambda_{2}(A)
$$

for all measurable $A$ would imply that $f^{-1}(y)$ is finite for every $y \in \mathbb{R}^{2}$.

Indeed, assume there is a point $y$ with $N$ preimages $x_{1}, x_{2}, \ldots, x_{N}$. Consider a radius $r$ so small that the balls $B_{r}\left(x_{i}\right)$ are disjoint. Denote by $A$ the disjoint union of $B_{r}\left(x_{i}\right)$ for $1 \leq i \leq N$. Since $f$ is $L$-Lipschitz, the image $f(A)$ is a subset of $B_{L r}(y)$. Together with (2.19), this implies $(L r)^{2} \geq \delta N r^{2}$, which is equivalent to $N \leq L^{2} / \delta$.

This means that if we find an independent way to prove the estimate (2.19) for any Lipschitz quotient mapping of the plane, this will imply the finiteness of point preimages and the regularity of the mapping. If this independent way of proving (2.19) works for higher dimensions, we will immediately get quasiregularity (in the sense of [7]) of Lipschitz quotient mappings from $\mathbb{R}^{n}$ to itself, which is now a challenging open question. 


\section{Ball noncollapsing functions}

THEOREM 3.1. The following statements are equivalent:

(i) there exists $f: \mathbb{R}^{1} \rightarrow \mathbb{R}^{1}, C$-BNC and L-Lipschitz, and a point $x \in \mathbb{R}^{1}$ such that $f^{-1}(x)$ is infinite;

(ii) $C / L<1 / 3$.

Proof. Let $C / L<1 / 3$. Without loss of generality we may assume that $L=1$ (the general case is obtained by rescaling). Then we find an $A>1$, such that $C=\left(1-A^{-2}\right) /\left(3-A^{-2}\right)$.

For an interval $I=[a, b]$ in $\mathbb{R}^{1}$ define the "hat function" $h_{I}(x)$ by $(b-a) / 2-\mid x-$ $(a+b) / 2 \mid$. Now let the mapping $\zeta_{A}: \mathbb{R}^{1} \rightarrow \mathbb{R}^{1}$ be defined by

$$
\zeta_{A}(x)= \begin{cases}x & \text { if } x \leq 0, \\ (-1)^{k} h_{\left[A^{-k}, A^{-k+1}\right]}(x) & \text { if } A^{-k} \leq x \leq A^{-k+1}, k>0 \text { integer, } \\ x-1 & \text { if } x>1 .\end{cases}
$$

Obviously, $\zeta_{A}$ is a 1-Lipschitz function (the simplest explanation is that its graph consists of line segments which form an angle of $45^{\circ}$ with the $x$-axis).

We will check now that $\zeta_{A}$ is BNC with constant $\left(1-A^{-2}\right) /\left(3-A^{-2}\right)$.

We reformulate this as the following lemma. Denote by $|I|$ the length of an interval $I$ in $\mathbb{R}^{1}$.

LeMmA 3.2. For any nonempty interval I in $\mathbb{R}^{1}$,

$$
\frac{\left|\zeta_{A}(I)\right|}{|I|} \geq \frac{1-A^{-2}}{3-A^{-2}}=C .
$$

Proof. Let

$$
\begin{gathered}
I_{1}=I \cap\left[-\frac{A-1}{2 A}, 1+\frac{A-1}{2 A^{2}}\right], \\
I_{2}=I \cap\left(-\infty,-\frac{A-1}{2 A}\right), \\
I_{3}=I \cap\left(1+\frac{A-1}{2 A^{2}}, \infty\right) .
\end{gathered}
$$

Since $\max _{[0,1]} \zeta_{A}=(A-1) / 2 A^{2}$, and $\min _{[0,1]} \zeta_{A}=-(A-1) / 2 A$, we conclude that for $i \neq j$ the intersection of $\zeta_{A}$-images of two intervals $I_{i}$ and $I_{j}$ is an empty set:

$$
\begin{aligned}
\zeta_{A} & >\frac{A-1}{2 A^{2}} & & \text { on }\left(1+\frac{A-1}{2 A^{2}}, \infty\right), \\
-\frac{A-1}{2 A} & \leq \zeta_{A} \leq \frac{A-1}{2 A^{2}} & & \text { on }\left[-\frac{A-1}{2 A}, 1+\frac{A-1}{2 A^{2}}\right], \\
\zeta_{A} & <-\frac{A-1}{2 A} & & \text { on }\left(-\infty,-\frac{A-1}{2 A}\right) .
\end{aligned}
$$

It is clear that $\left|\zeta_{A}\left(I_{j}\right)\right|=\left|I_{j}\right|$ for $j=2,3$. Thus, it is enough to show that $\left|\zeta_{A}\left(I_{1}\right)\right| \geq C\left|I_{1}\right|$. We will assume $\left|I_{1}\right| \neq 0$, otherwise this inequality is obvious. 
Let $I_{1}=\left[a_{1}, b_{1}\right]$ and $J=I_{1} \cap[0,1]=[a, b]$.

In what follows, we use the fact that if $[s, t]$ is contained in an interval of the form $\left[A^{-(k+1)}, A^{-k}\right]$, then $\left|\zeta_{A}([s, t])\right| \geq 1 / 2|[s, t]|$ by the property of the hat function $h_{\left[A^{-(k+1)}, A^{-k}\right]}$. Case 1. If both $a$ and $b$ are contained in the same interval of the form $\left[A^{-(k+1)}, A^{-k}\right]$, then $\left|\zeta_{A}([a, b])\right| \geq(1 / 2)|[a, b]|$.

If $J=I_{1}$, this finishes the proof since $1 / 2>C$.

If $J \neq I_{1}$, then the only possibility is that $b_{1}>b=1$ and $a_{1}=a \in[1 / A, 1]$. Then $\zeta_{A}$ is negative on $[a, b]$ and is positive on $\left[b, b_{1}\right]$, so $\left|\zeta_{A}\left(I_{1}\right)\right|=\left|\zeta_{A}([a, b])\right|+\left(b_{1}-b\right) \geq(1 / 2)\left|I_{1}\right|>$ $C\left|I_{1}\right|$.

Case 2. If $a$ and $b$ are contained in two adjacent intervals of the form $\left[A^{-(k+1)}, A^{-k}\right]$, then since $\zeta_{A}$ has different signs on those two intervals, we conclude that $\zeta_{A}(J) \geq 1 / 2|J|>C|J|$.

If $J \neq I_{1}$, then necessarily $b_{1}>b=1$ and $a_{1}=a \in\left[1 / A^{2}, 1 / A\right]$. Let $x=1 / A-a$ and $y=$ $b_{1}-1$. By the construction of the interval $I_{1}, y \leq(A-1) / 2 A^{2}=\max _{\left[A^{-2}, A^{-1}\right]} \zeta_{A}$. Hence

$$
\max _{\left[a_{1}, b_{1}\right]} \zeta_{A}=\min \left(\frac{A-1}{2 A^{2}}, \max \{x, y\}\right)
$$

Denote this number by $\alpha$. Then

$$
\begin{gathered}
\left|\zeta_{A}\left(I_{1}\right)\right|=\frac{1}{2}\left(1-\frac{1}{A}\right)+\alpha \geq \frac{1}{2}\left(\left(1-\frac{1}{A}\right)+x+y\right) \\
=\frac{1}{2}\left|I_{1}\right|>C\left|I_{1}\right| \quad \text { if } \alpha=\max \{x, y\}, \\
\left|\zeta_{A}\left(I_{1}\right)\right|=\frac{1}{2}\left(1-\frac{1}{A}\right)+\frac{A-1}{2 A^{2}}=\frac{1}{2}-\frac{1}{2 A^{2}} \geq \frac{1}{3}+\frac{1}{6 A}-\frac{1}{2 A^{2}} \\
=\frac{1}{3}\left(1+\frac{A-1}{2 A^{2}}-\frac{1}{A^{2}}\right) \geq \frac{1}{3}\left|I_{1}\right|>C\left|I_{1}\right| \quad \text { otherwise }
\end{gathered}
$$

(since $\left.I_{1} \subset\left[1 / A^{2}, 1+(A-1) / 2 A^{2}\right],\left|I_{1}\right| \leq 1+(A-1) / 2 A^{2}-1 / A^{2}\right)$.

Case 3. If $J$ overlaps with three intervals of the form $\left[A^{-(k+1)}, A^{-k}\right]$, then it must contain one of them. Assume

$$
\frac{1}{A^{n+3}} \leq a \leq \frac{1}{A^{n+2}}<\frac{1}{A^{n+1}} \leq b \leq \frac{1}{A^{n}}
$$

If $b=1$, then $n=0$, so $a \in\left[1 / A^{3}, 1 / A^{2}\right]$. Then

$$
\zeta_{A}(J)=\left[-\frac{1}{2} \frac{A-1}{A}, \frac{1}{2} \frac{A-1}{A^{2}}\right]
$$

therefore $\zeta_{A}\left(I_{1}\right)=\zeta_{A}(J)$ (even if $I_{1} \neq J$; the right-hand side is simply the image of the whole interval $\left[-(A-1) / 2 A, 1+(A-1) / 2 A^{2}\right]$ containing $\left.I_{1}\right)$, and

$$
\left|\zeta_{A}\left(I_{1}\right)\right|=\frac{1}{2}\left(\frac{A-1}{A}+\frac{A-1}{A^{2}}\right)=\frac{A^{2}-1}{2 A^{2}} .
$$


At the same time

$$
\left|I_{1}\right| \leq 1+\frac{A-1}{2 A^{2}}-\left(-\frac{A-1}{2 A}\right)=\frac{3 A^{2}-1}{2 A^{2}},
$$

so, $\left|\zeta_{A}\left(I_{1}\right)\right| \geq\left(A^{2}-1\right) /\left(3 A^{2}-1\right) \cdot\left|I_{1}\right|$.

If, however, $b<1$, then $I_{1}=J$. Without loss of generality we may assume that $\zeta_{A}>0$ on the interval $\left(1 / A^{n+2}, 1 / A^{n+1}\right)$, which is contained in $J=I_{1}=[a, b]$.

Let $x=1 / A^{n+2}-a$ and $y=b-1 / A^{n+1}$. It is clear that $\max _{[a, b]} \zeta_{A}=(A-1) / 2 A^{n+2}$.

(1) If $y \geq(A-1) / 2 A^{n+1}$, then $\min _{[a, b]} \zeta_{A}=-(A-1) / 2 A^{n+1}$, and so

$$
\left|\zeta_{A}\left(I_{1}\right)\right|=\frac{A-1}{2 A^{n+2}}+\frac{A-1}{2 A^{n+1}}=\frac{A^{2}-1}{2 A^{n+2}}
$$

and $\left|I_{1}\right| \leq 1 / A^{n}$. We see that $\left|\zeta_{A}\left(I_{1}\right)\right| /\left|I_{1}\right| \geq\left(A^{2}-1\right) / 2 A^{2} \geq\left(A^{2}-1\right) /\left(3 A^{2}-1\right)$ (since $A>1)$.

(2) If $x \leq y<(A-1) / 2 A^{n+1}$, then $\min _{[a, b]} \zeta_{A}=-y$, therefore

$$
\left|\zeta_{A}\left(I_{1}\right)\right|=\frac{A-1}{2 A^{n+2}}+y \geq \frac{1}{2}\left(\frac{A-1}{A^{n+2}}+x+y\right)=\frac{1}{2}\left|I_{1}\right|>C\left|I_{1}\right| .
$$

(3) If $y<x \leq(A-1) / 2 A^{n+3}$ we get $\min _{[a, b]} \zeta_{A}=-x$, and

$$
\left|\zeta_{A}\left(I_{1}\right)\right|=\frac{A-1}{2 A^{n+2}}+x \geq \frac{1}{2}\left(\frac{A-1}{A^{n+2}}+x+y\right)=\frac{1}{2}\left|I_{1}\right| .
$$

(4) If $y<(A-1) / 2 A^{n+1}, y<x$, and $x>(A-1) / 2 A^{n+3}$, then $\min _{[a, b]} \zeta_{A}=-(A-1) / 2 A^{n+3}$. In this case

$$
\begin{gathered}
\left|\zeta_{A}\left(I_{1}\right)\right|=\frac{A-1}{2 A^{n+2}}+\frac{A-1}{2 A^{n+3}}, \\
\left|I_{1}\right|=\frac{A-1}{A^{n+2}}+x+y .
\end{gathered}
$$

We check that

$$
\left|\zeta_{A}\left(I_{1}\right)\right| \geq \frac{1}{3}\left(\frac{A-1}{A^{n+2}}+x+y\right)
$$

Since $y<x \leq(A-1) / A^{n+3}$, we conclude that the right-hand side is not greater than $(A-1) / 3 A^{n+3}(A+2)$. The left-hand side is equal to $(A-1) / 2 A^{n+3}(A+1)$. So it is enough to check that $(A+2) / 3 \leq(A+1) / 2$, which is true for $A>1$.

Case 4. If $J=[a, b]$ overlaps with at least four intervals of the form $\left[A^{-(k+1)}, A^{-k}\right]$, then we consider four possibilities.

(4A) $a=0$ and $b=1$. In this case $\left|I_{1}\right| \leq 1+(A-1) / 2 A+(A-1) / 2 A^{2}=\left(3 A^{2}-1\right) / 2 A^{2}$ and $\left|\zeta_{A}\left(I_{1}\right)\right|=(A-1) / 2 A+(A-1) / 2 A^{2}=\left(A^{2}-1\right) / 2 A^{2}$. So, $\left|\zeta_{A}\left(I_{1}\right)\right| /\left|I_{1}\right| \geq\left(A^{2}-1\right) /$ $\left(3 A^{2}-1\right)$.

(4B) $a>0$ and $b=1$. In this case $\zeta_{A}(J)=\zeta_{A}[a, 1]=\zeta_{A}[0,1]$, since the minimum of $\zeta_{A}$ on $[a, 1]$ is attained at the point $(1 / 2)(1 / A+1)$ and the maximum-at $(1 / 2)\left(1 / A^{2}+1 / A\right)$. So, $\left|\zeta_{A}\left(I_{1}\right)\right|=\left|\zeta_{A}\left[0, b_{1}\right]\right| \geq\left(A^{2}-1\right) /\left(3 A^{2}-1\right)\left|\left[0, b_{1}\right]\right| \geq\left(A^{2}-1\right) /\left(3 A^{2}-1\right)\left|I_{1}\right|$. 
(4C) $a>0$ and $b<1$. This case follows from the next one, (4D), in the same way as (4B) follows from $(4 \mathrm{~A})$ : we use that $I_{1}=J$ and $\left|\zeta_{A}\left(I_{1}\right)\right|=\zeta_{A}|[0, b]|$.

(4D) $a=0$ and $b<1$. Assume $\zeta_{A}(b) \leq 0$ and $b \in\left[1 / A^{n+1}, 1 / A^{n}\right]$. (The case $\zeta_{A}(b)>0$ will be treated later in $(4 \mathrm{D}-\mathrm{V})$ and $(4 \mathrm{D}-\mathrm{VI})$.) Then

$$
\max _{J} \zeta_{A}=\max _{I_{1}} \zeta_{A}=\frac{1}{2}\left(\frac{1}{A^{n+1}}-\frac{1}{A^{n+2}}\right) .
$$

Denote $x=-a_{1}$ and $y=b-1 / A^{n+1}$.

(4D-I) $x \geq(A-1) / 2 A^{n+1}$. Then $\min _{I_{1}} \zeta_{A}=-x$. So,

$$
\left|\zeta_{A}\left(I_{1}\right)\right|=x+\frac{A-1}{2 A^{n+2}}
$$

and $\left|I_{1}\right| \leq x+1 / A^{n}$.

In order to prove $\left|\zeta_{A}\left(I_{1}\right)\right| \geq C\left|I_{1}\right|$, it suffices to show that

$$
x+\frac{A-1}{2 A^{n+2}} \geq \frac{A^{2}-1}{3 A^{2}-1}\left(x+\frac{1}{A^{n}}\right) .
$$

This is equivalent to

$$
x\left(1-\frac{A^{2}-1}{3 A^{2}-1}\right) \geq \frac{A^{2}-1}{3 A^{2}-1} \cdot \frac{1}{A^{n}}-\frac{A-1}{2 A^{n+2}} .
$$

Rewriting this inequality, we get

$$
x \frac{2 A^{2}}{3 A^{2}-1} \geq \frac{A-1}{\left(3 A^{2}-1\right) 2 A^{n+2}}\left[2 A^{3}-A^{2}+1\right] .
$$

Since $x \geq(A-1) / 2 A^{n+1}$, the left-hand side is at least $\left((A-1) / 2 A^{n+1}\right) \cdot\left(2 A^{2} /\left(3 A^{2}-1\right)\right)$, so it is enough to prove that

$$
2 A^{2} \geq \frac{1}{A}\left[2 A^{3}-A^{2}+1\right]
$$

which is true for $A>1$.

(4D-II) $x<(A-1) / 2 A^{n+1}, y \geq(A-1) / 2 A^{n+1}$. Then $\min _{I_{1}} \zeta_{A}=-(A-1) / 2 A^{n+1}$. Therefore,

$$
\left|\zeta_{A}\left(I_{1}\right)\right|=\frac{A-1}{2 A^{n+2}}+\frac{A-1}{2 A^{n+1}}=\frac{A^{2}-1}{2 A^{n+2}},
$$

and $\left|I_{1}\right| \leq x+1 / A^{n}$. We want to show that

$$
\frac{A^{2}-1}{2 A^{n+2}} \geq \frac{A^{2}-1}{3 A^{2}-1}\left(x+\frac{1}{A^{n}}\right),
$$

which is equivalent to

$$
\frac{1}{2 A^{n+2}} \geq \frac{1}{3 A^{2}-1}\left(x+\frac{1}{A^{n}}\right) .
$$


Since $x<(A-1) / 2 A^{n+1}$, the right-hand side is less than or equal to

$$
\frac{1}{3 A^{2}-1} \cdot \frac{3 A-1}{2 A^{n+1}}=\frac{1}{2 A^{n+2}}\left(\frac{3 A^{2}-A}{3 A^{2}-1}\right) \leq \frac{1}{2 A^{n+2}},
$$

since $A>1$.

(4D-III) $x, y<(A-1) / 2 A^{n+1}$, and $\max \{x, y\} \geq(A-1) / 2 A^{n+3}$. Then $\min _{I_{1}} \zeta_{A}=-\max \{x$, $y\}$, so $\left|\zeta_{A}\left(I_{1}\right)\right|=\max \{x, y\}+(A-1) / 2 A^{n+2}$ and $\left|I_{1}\right|=x+y+1 / A^{n+1}$.

We want to show that

$$
\max \{x, y\}+\frac{A-1}{2 A^{n+2}} \geq \frac{A^{2}-1}{3 A^{2}-1}\left(x+y+\frac{1}{A^{n+1}}\right) .
$$

Replacing $x+y$ by $2 \max \{x, y\}$, we get a stronger inequality

$$
\max \{x, y\}\left(1-2 \frac{A^{2}-1}{3 A^{2}-1}\right) \geq \frac{A^{2}-1}{3 A^{2}-1} \cdot \frac{1}{A^{n+1}}-\frac{A-1}{2 A^{n+2}},
$$

which is equivalent to

$$
\max \{x, y\} \frac{A^{2}+1}{3 A^{2}-1} \geq \frac{A-1}{\left(3 A^{2}-1\right) 2 A^{n+2}}\left(2 A^{2}+2 A-3 A^{2}+1\right) .
$$

Since $\max \{x, y\} \geq(A-1) / 2 A^{n+3}$, it is enough to check that

$$
\frac{A-1}{2 A^{n+3}} \cdot\left(A^{2}+1\right) \geq \frac{A-1}{2 A^{n+2}}\left(-A^{2}+2 A+1\right) .
$$

The latter inequality is equivalent to $A^{2}+1 \geq A\left(-A^{2}+2 A+1\right)$, which is the same as $A^{3}-$ $A^{2} \geq A-1$, true for $A>1$.

(4D-IV) $\max \{x, y\}<(A-1) / 2 A^{n+3}$. Then $\min _{I_{1}} \zeta_{A}=-(A-1) / 2 A^{n+3}$, so $\left|\zeta_{A}\left(I_{1}\right)\right|=$ $(A-1) / 2 A^{n+3}+(A-1) / 2 A^{n+2}$ and

$$
\left|I_{1}\right|=x+y+\frac{1}{A^{n+1}} \leq 2 \cdot \frac{1}{2} \frac{A-1}{A^{n+3}}+\frac{1}{A^{n+1}} .
$$

The inequality $\left|\zeta_{A}\left(I_{1}\right)\right| \geq\left(A^{2}-1\right) /\left(3 A^{2}-1\right)\left|I_{1}\right|$ then becomes a particular case of (4D-III) $\left(x=y=(A-1) / 2 A^{n+3}\right)$.

This finishes the proof of (4D) under the assumption $\zeta_{A}(b) \leq 0$.

Now assume $\zeta_{A}(b)>0$.

In order to determine the maximum and minimum values of $\zeta_{A}$ on the interval $I_{1}$, we must compare $x$ with $(A-1) / 2 A^{n+2}$, and $y$ with $(A-1) / 2 A^{n+1}$.

$(4 \mathrm{D}-\mathrm{V}) \quad y \geq(A-1) / 2 A^{n+1}$. Then one has $\min _{I_{1}} \zeta_{A}=-\max \left\{x,(A-1) / 2 A^{n+2}\right\}$ and $\max _{I_{1}} \zeta_{A}=(A-1) / 2 A^{n+1}$. So $\left|\zeta_{A}\left(I_{1}\right)\right|=\max \left\{x,(A-1) / 2 A^{n+2}\right\}+(A-1) / 2 A^{n+1}$, and

$$
\left|I_{1}\right| \leq x+\frac{1}{A^{n}} \leq \max \left\{x, \frac{A-1}{2 A^{n+2}}\right\}+\frac{1}{A^{n}} .
$$

It is enough to prove that

$$
\alpha+\frac{A-1}{2 A^{n+1}} \geq \frac{A^{2}-1}{3 A^{2}-1}\left(\alpha+\frac{1}{A^{n}}\right),
$$


where $\alpha=\max \left\{x,(A-1) / 2 A^{n+2}\right\}$. This inequality is equivalent to

$$
\begin{aligned}
\alpha \frac{2 A^{2}}{3 A^{2}-1} & \geq \frac{A-1}{2\left(3 A^{2}-1\right) A^{n+1}}\left(-3 A^{2}+1+2 A(A+1)\right) \\
& =\frac{A-1}{2\left(3 A^{2}-1\right) A^{n+1}}\left(-A^{2}+2 A+1\right) .
\end{aligned}
$$

Since $\alpha \geq(A-1) / 2 A^{n+2}$, it is enough to prove that

$$
\frac{A-1}{\left(3 A^{2}-1\right) A^{n}} \geq \frac{A-1}{2\left(3 A^{2}-1\right) A^{n+1}}\left(-A^{2}+2 A+1\right) .
$$

The latter inequality is the same as $1 \geq(1 / 2 A)\left(-A^{2}+2 A+1\right)$; this is always true for $A>1$.

$(4 \mathrm{D}-\mathrm{VI}) \quad y<(A-1) / 2 A^{n+1}$. In this case $\left|\zeta_{A}\left(I_{1}\right)\right|=\max \left\{x,(A-1) / 2 A^{n+2}\right\}+\max \{y$, $\left.(A-1) / 2 A^{n+3}\right\}$ and $\left|I_{1}\right|=x+y+1 / A^{n+1} \leq \max \left\{x,(A-1) / 2 A^{n+2}\right\}+\max \{y,(A-1) /$ $\left.2 A^{n+3}\right\}+1 / A^{n+1}$.

We want to prove that $\alpha+\beta \geq\left(\left(A^{2}-1\right) /\left(3 A^{2}-1\right)\right)\left(\alpha+\beta+1 / A^{n+1}\right)$, where $\alpha=\max \{x$, $\left.(A-1) / 2 A^{n+2}\right\}$ and $\beta=\max \left\{y,(A-1) / 2 A^{n+3}\right\}$. The latter inequality is equivalent to

$$
(\alpha+\beta) \frac{2 A^{2}}{3 A^{2}-1} \geq \frac{A^{2}-1}{\left(3 A^{2}-1\right) A^{n+1}} .
$$

But $\alpha+\beta \geq(A-1) / 2 A^{n+2}+(A-1) / 2 A^{n+3}=\left(A^{2}-1\right) / 2 A^{n+3}$, so the inequality follows.

Now we prove that the bound of $1 / 3$ cannot be improved, that is, if a Lipschitz and $\mathrm{BNC}$ mapping has infinite point preimages, then the ratio of constants $C / L$ is strictly less than $1 / 3$.

We assume again that the Lipschitz constant of the mapping is 1 , clearly without loss of generality. We denote by $|I|$ the length of an interval $I \subset \mathbb{R}$.

Note that for a continuous function from $\mathbb{R}$ to $\mathbb{R}$ the constant $C$ does not exceed the lower bound of $|f(I)| /|I|$, taken over all intervals $I \subset \mathbb{R}$.

Lemma 3.3. Suppose $f$ is a 1-Lipschitz function on $\mathbb{R}$, and $0=x_{0}<x_{1}<x_{2}<\cdots<x_{n}$ are roots of $f$ (meaning $f\left(x_{i}\right)=0$ ). Then

$$
\left|f\left[0, x_{n}\right]\right| \leq \frac{1}{2} \max _{0 \leq j<k \leq n-1}\left(x_{j+1}-x_{j}\right)+\left(x_{k+1}-x_{k}\right) .
$$

Proof. Denote $I_{s}=\left[x_{s}, x_{s+1}\right]$ and assume that $I_{k}$ and $I_{j}$ are the longest and second longest intervals, respectively, among all $I_{s}, 0 \leq s \leq n-1$. Then $f\left(I_{s}\right) \subset\left[-\left|I_{j}\right| / 2,\left|I_{j}\right| / 2\right]$ for all $s \neq k$, since $f$ is 1-Lipschitz, $f$ vanishes at the endpoints of these intervals and $\left|I_{s}\right| \leq\left|I_{j}\right|$. 
But $f\left(I_{k}\right)$ is an interval of length at most $\left|I_{k}\right| / 2$, containing zero, therefore

$$
\left|f\left(I_{k}\right) \backslash\left[-\frac{\left|I_{j}\right|}{2}, \frac{\left|I_{j}\right|}{2}\right]\right| \leq \frac{\left(\left|I_{k}\right|-\left|I_{j}\right|\right)}{2} .
$$

Thus, $\left|f\left[0, x_{n}\right]\right| \leq\left|I_{j}\right|+\left(\left|I_{k}\right|-\left|I_{j}\right|\right) / 2=\left(\left|I_{k}\right|+\left|I_{j}\right|\right) / 2$.

Corollary 3.4. If $I=\left[0, x_{n}\right]$ is an interval as in Lemma 3.3 with $n \geq 3$ (i.e., an interval containing at least two roots of $f$ other than its endpoints), and there exists an interval $I_{1} \supset I$ such that $\left|I_{1}\right|=|I|+|f(I)|$ and $f\left(I_{1}\right)=f(I)$, then $C<1 / 3$.

Proof. As we noted in the beginning, $C \leq\left|f\left(I_{1}\right)\right| /\left|I_{1}\right|=|f(I)| /(|I|+|f(I)|)$. Lemma 3.3 implies that $|f(I)|<|I| / 2$, then $|f(I)| /(|I|+|f(I)|)$ is strictly less than $1 / 3$.

We return to the proof of the theorem.

We will assume that $f(0)=0$ and the point 0 has an infinite preimage under $f$, and we will show that $C<1 / 3$ in such a case.

Throughout the proof, we will use the following notation. Let $t$ be a nonnegative number. Denote

$$
\begin{gathered}
a(t)=\sup f[0, t], \\
b(t)=-\inf f[0, t], \\
n_{a}(t)=\inf \left\{t^{\prime}>t: a\left(t^{\prime}\right)>a(t)\right\}, \\
n_{b}(t)=\inf \left\{t^{\prime}>t: b\left(t^{\prime}\right)>b(t)\right\}, \\
m_{a}(t)=\sup \left\{t^{\prime} \in\left[t, n_{b}(t)\right]: f\left(t^{\prime}\right)=\max f\left[t, n_{b}(t)\right]\right\}, \\
m_{b}(t)=\sup \left\{t^{\prime} \in\left[t, n_{a}(t)\right]: f\left(t^{\prime}\right)=\min f\left[t, n_{a}(t)\right]\right\} .
\end{gathered}
$$

Note that if $a(t)$ and $b(t)$ are both positive, then $n_{a}(t) \neq n_{b}(t)$ since $f$ is continuous.

Suppose that $M=\{x: f(x)=0\}$ is an infinite set.

Case 1. $M$ is unbounded.

This case may be split into two: if $M$ is, say, bounded from below (but unbounded from above), we assume that

$$
\begin{gathered}
\exists\left\{x_{n}\right\}: x_{n}>0, x_{n} \longrightarrow+\infty, f\left(x_{n}\right)=f(0)=0, \\
f \text { has no roots in }(-\infty, 0) ;
\end{gathered}
$$

if $M$ is unbounded both from above and from below, we may assume that

$$
\begin{aligned}
& \exists\left\{x_{n}\right\}: x_{n}>0, x_{n} \longrightarrow+\infty, f\left(x_{n}\right)=f(0)=0, \\
& \exists\left\{y_{n}\right\}: y_{n}<0, y_{n} \longrightarrow-\infty, f\left(y_{n}\right)=f(0)=0 .
\end{aligned}
$$

Now we discuss the case where $M$ is unbounded from above and bounded from below. We prove that $C<1 / 3$ in this case in Lemmas 3.5 and 3.6.

Lemma 3.5. If $f: \mathbb{R} \rightarrow \mathbb{R}$ is 1-Lipschitz, C-ball noncollapsing, (3.39) holds and the image of $[0,+\infty)$ is not the whole real line, then $C<1 / 3$. 
Proof. If $f[0,+\infty)$ is a bounded interval, then $\inf |f(I)| /|I|$ over all intervals $I \subset \mathbb{R}$ is equal to zero, thus $C=0<1 / 3$.

Otherwise $f[0,+\infty)$ is a ray. Assume that inf $f[0,+\infty)=-m$ is finite and $\sup f[0,+\infty)$ $=+\infty$. Note first that $m \geq 0$, since $f(0)=0$. Without loss of generality we may assume that $x_{n+1}>x_{n}$, and moreover, $x_{n+1}-x_{n}>x_{n}-x_{n-1}$, for each $n$. Then Lemma 3.3 implies that for each $n \geq 2$ one has

$$
a\left(x_{n}\right)+b\left(x_{n}\right)=\left|f\left[0, x_{n}\right]\right| \leq \frac{\left(x_{n}-x_{n-2}\right)}{2} .
$$

Consider $n \geq 3$ such that $\left|f\left[0, x_{n}\right]\right|>3 m$ and

$$
x_{n-2}>3 m \text {. }
$$

Let $a=a\left(x_{n}\right)$ and $b=b\left(x_{n}\right)$. Then

$$
a=(a+b)+(m-b)-m>3 m+0-m=2 m \geq b .
$$

We show that $\left|f\left[-b, x_{n}+a\right]\right| \leq a+m$.

Indeed,

$$
f(x) \in[-b, b] \subset[-m, a] \quad \text { for } x \in[-b, 0],
$$

since

$$
\begin{gathered}
m \geq b, \quad b<a, \quad f(0)=0, \quad f \text { is 1-Lipschitz; } \\
f(x) \in[-m, a] \quad \text { for } x \in\left[x_{n}, x_{n}+a\right]
\end{gathered}
$$

since

$$
\left.f\right|_{[0,+\infty)} \geq-m, \quad f\left(x_{n}\right)=0, \quad f \text { is 1-Lipschitz. }
$$

Then $f\left[-b, x_{n}+a\right] \subset[-m, a]$, so $\left|f\left[-b, x_{n}+a\right]\right| \leq m+a$.

Then

$$
C \leq \frac{\left|f\left[-b, x_{n}+a\right]\right|}{\left|\left[-b, x_{n}+a\right]\right|} \leq \frac{m+a+b}{a+b+x_{n}}=1-\frac{x_{n}-m}{a+b+x_{n}} \text {. }
$$

By (3.42), $a+b \leq\left(x_{n}-x_{n-2}\right) / 2$, so we have

$$
1-\frac{x_{n}-m}{a+b+x_{n}} \leq 1-\frac{x_{n}-m}{\left(x_{n}-x_{n-2}\right) / 2+x_{n}}
$$


since $m<x_{n}$ by (3.43). The latter is strictly less than $1 / 3$ :

$$
1-\frac{x_{n}-m}{\left(x_{n}-x_{n-2}\right) / 2+x_{n}}<1-\frac{x_{n}-x_{n-2} / 3}{\left(x_{n}-x_{n-2}\right) / 2+x_{n}}=\frac{1}{3} \quad \text { by (3.43) }
$$

Together with (3.48) this gives $C<1 / 3$.

Lemma 3.6. If $f: \mathbb{R} \rightarrow \mathbb{R}$ is 1 -Lipschitz, C-ball noncollapsing, (3.40) holds and $f[0,+\infty)=$ $(-\infty,+\infty)$, then $C<1 / 3$.

Proof. Since $f$ has no roots in $(-\infty, 0)$, its sign there is constant. Assume, for example, that $f$ is strictly positive on $(-\infty, 0)$.

Find a root $x$ of $f$ such that $a(x)>0$ and $b(x)>0$, and the interval $(0, x)$ contains at least two roots of $f$ (since $M$ is unbounded, this is possible).

Then

$$
f[-a(x), 0] \subset[0, a(x)] \subset[-b(x), a(x)]
$$

since $f$ is nonnegative on $[-a(x), 0], f(0)=0$ and $f$ is 1-Lipschitz.

If $b(x) \leq a(x)$, then

$$
f[x, x+b(x)] \subset[-b(x), a(x)]
$$

since $f$ is 1-Lipschitz, $f(x)=0$ and so $f[x, x+b(x)] \subset[-b(x), b(x)] \subset[-b(x), a(x)]$.

Together (3.51) and (3.52) imply $f[-a(x), x+b(x)]=f[0, x]$, therefore $C<1 / 3$, by Corollary 3.4 applied to the interval $I=[0, x]$.

Assume now $b(x)>a(x)$. We want to show that (3.52) still holds, then we would be able to conclude that $C<1 / 3$.

Note that since the $f$-image of $(0,+\infty)$ is the whole real line, $n_{a}(x)$ and $n_{b}(x)$ are finite numbers.

If $n_{b}(x)<n_{a}(x)$, then (3.52) holds since $x+b(x) \leq n_{b}(x)$ and by definition of $n_{a}(x)$ one has $f(t) \leq a$ for all $t \in\left[0, n_{a}(x)\right]$.

If $n_{a}(x)<n_{b}(x)$, then $f$ has roots in the interval $\left(x, n_{b}(x)\right)$, since $f\left(n_{a}(x)\right)=a(x)>0$ and $f\left(n_{b}(x)\right)=-b(x)<0$.

Let $z_{0}=\sup \left\{z \in\left(x, n_{b}(x)\right): f(z)=0\right\}$. Consider then the interval $\left[0, z_{0}\right]$ between the two roots. Note that $b\left(z_{0}\right)=b(x)$, and thus $n_{b}\left(z_{0}\right)=n_{b}(x)$. At the same time $a\left(z_{0}\right)>a(x)$.

If it turns out that $b\left(z_{0}\right) \leq a\left(z_{0}\right)$, then (3.51) and (3.52) hold if we substitute $z_{0}$ instead of $x$, so we immediately get $C<1 / 3$. If, on the contrary, $b\left(z_{0}\right)>a\left(z_{0}\right)$, then $n_{b}\left(z_{0}\right)<n_{a}\left(z_{0}\right)$ (otherwise $f$ would have roots between $z_{0}$ and $n_{b}\left(z_{0}\right)$ ). We now replace $x$ by $z_{0}$ and get back to the case $n_{b}(x)<n_{a}(x)$, where, as we have already shown, $C<1 / 3$.

Thus we have proved that if (3.39) and (3.40) hold (i.e., $M$ is bounded from below), the statement of the Theorem is true. We now discuss why $C<1 / 3$ in case (3.41) takes place.

By Lemma 3.5, if the image of either $[0,+\infty)$ or $(-\infty, 0]$ is not the whole line, then $C<1 / 3$. Thus we may assume that $f[0,+\infty)=f(-\infty, 0]=(-\infty,+\infty)$. 
Lemma 3.7. If $f: \mathbb{R} \rightarrow \mathbb{R}$ is 1-Lipschitz, and (3.39) holds, then there exists a sequence $p_{1}, p_{2}, \ldots$ of points such that

$$
\begin{gathered}
0<p_{k}<p_{k+1} \quad \forall k \geq 1, \\
f\left(p_{1}\right)>0, \quad f\left(p_{k}\right) f\left(p_{k+1}\right)<0 \quad \forall k \geq 1, \\
\left|f\left(p_{k}\right)\right|<\left|f\left(p_{k+2}\right)\right| \quad \forall k \geq 1, \\
f\left(p_{k}\right)=a\left(p_{k}\right) \quad \text { if } f\left(p_{k}\right)>0, \quad f\left(p_{k}\right)=-b\left(p_{k}\right) \quad \text { if } f\left(p_{k}\right)<0, \\
f(x) \in\left[f\left(p_{k}\right), f\left(p_{k+1}\right)\right] \quad \text { or }\left[f\left(p_{k+1}\right), f\left(p_{k}\right)\right] \quad \text { for } x \in\left[p_{k}, p_{k+1}\right] .
\end{gathered}
$$

Remark 3.8. Since $f[0,+\infty)=(-\infty,+\infty),(3.56)$ implies

$$
\left|f\left(p_{k}\right)\right| \rightarrow \infty \text {. }
$$

Proof of Lemma 3.7. Consider $n$ such that $a=a\left(x_{n}\right)>0$ and $b=b\left(x_{n}\right)>0$. Let $z_{1}=\sup \{z$ $\left.\in\left[0, x_{n}\right]: f(z)=a\right\}$ and $z_{2}=\sup \left\{z \in\left[0, x_{n}\right]: f(z)=-b\right\}$. Without loss of generality (since we may replace $f$ by $-f$ ) assume that $z_{1}<z_{2}$. Then put $p_{1}=z_{1}$ and $p_{2}=m_{b}\left(z_{1}\right)$. We check conditions (3.53)-(3.57) for $p_{1}$ and $p_{2}$.

(i) Condition (3.53). We know that $p_{2}=m_{b}\left(p_{1}\right) \geq p_{1}$, but since $z_{1}<z_{2} \leq x_{n} \leq n_{a}\left(z_{1}\right)$,

$$
f\left(p_{2}\right)=\min f\left[z_{1}, n_{a}\left(z_{1}\right)\right] \leq f\left(z_{2}\right)=-b<a=f\left(p_{1}\right),
$$

so $p_{2} \neq p_{1}$.

(ii) Condition (3.54). Since $f\left(p_{1}\right)=a>0$ and $f\left(p_{2}\right) \leq-b<0$, we get $f\left(p_{1}\right) f\left(p_{2}\right)<$ 0 .

(iii) Condition (3.55). There is nothing to check.

(iv) Condition (3.56). $f\left(p_{1}\right)=a=a\left(p_{1}\right), f\left(p_{2}\right) \leq-b$, so $f\left(p_{2}\right)=-b\left(p_{2}\right)$.

(v) Condition (3.57). If $x \in\left[p_{1}, p_{2}\right]$, then $f(x) \geq \min f\left[p_{1}, p_{2}\right] \geq \min f\left[p_{1}, n_{a}\left(p_{1}\right)\right]=$ $f\left(p_{2}\right)$ and $f(x) \leq f\left(p_{1}\right)=a\left(p_{1}\right)$, since $x \leq p_{2} \leq n_{a}\left(p_{1}\right)$.

Assume now that $p_{1}<\cdots<p_{n-1}<p_{n}$ are constructed. Then let

$$
p_{n+1}= \begin{cases}m_{b}\left(p_{n}\right) & \text { if } f\left(p_{n}\right)>0, \\ m_{a}\left(p_{n}\right) & \text { if } f\left(p_{n}\right)<0 .\end{cases}
$$

Since we know that the image of $[0,+\infty)$ is the whole line, we conclude that $p_{n+1}$ is a finite number.

We check conditions (3.53)-(3.57) for $p_{n+1}$. Assume, for example, that $f\left(p_{n}\right)>0$ (so that $\left.p_{n}=m_{a}\left(p_{n-1}\right)\right)$.

(i) Condition (3.53). Firstly we show that $n_{a}\left(p_{n}\right)>n_{b}\left(p_{n}\right)$. Indeed, $n_{b}\left(p_{n}\right)=n_{b}\left(p_{n-1}\right)$, and $p_{n}$ is the rightmost point in $\left[p_{n-1}, n_{b}\left(p_{n-1}\right)\right]$ where $f$ attains the maximum value on $\left[p_{n-1}, n_{b}\left(p_{n-1}\right)\right]$, so $n_{a}\left(p_{n}\right)$ cannot be inside this interval. This means $n_{a}\left(p_{n}\right)>n_{b}\left(p_{n-1}\right)=n_{b}\left(p_{n}\right)$. Now, $p_{n+1}=m_{b}\left(p_{n}\right) \geq p_{n}$, and

$$
f\left(p_{n+1}\right)=\min f\left[p_{n}, n_{a}\left(p_{n}\right)\right] \leq \min f\left[p_{n}, n_{b}\left(p_{n}\right)\right]<0, \quad \text { so } p_{n+1} \neq p_{n} .
$$

(ii) Condition (3.54). Since $f\left(p_{n}\right)>0$ and $f\left(p_{n+1}\right)<0$, we get $f\left(p_{n}\right) f\left(p_{n+1}\right)<0$. 
(iii) Condition (3.55). The inequality $n_{a}\left(p_{n}\right)>n_{b}\left(p_{n-1}\right)$ implies that $f$ takes values less than $-b\left(p_{n-1}\right)$ in the interval $\left[p_{n}, n_{a}\left(p_{n}\right)\right]$. Since $f\left(p_{n+1}\right)$ is the least value of $f$ in $\left[p_{n}, n_{a}\left(p_{n}\right)\right]$, we have

$$
f\left(p_{n+1}\right)<-b\left(p_{n-1}\right)=f\left(p_{n-1}\right) .
$$

(iv) Condition (3.56). Since $\min f\left[p_{n}, n_{a}\left(p_{n}\right)\right]=f\left(p_{n+1}\right)<f\left(p_{n-1}\right)=\min f\left[0, p_{n}\right], f$ attains its minimum on $\left[0, n_{a}\left(p_{n}\right)\right] \supset\left[0, p_{n+1}\right]$ at $p_{n+1}$.

(v) Condition (3.57). If $x \in\left[p_{n}, p_{n+1}\right]$, then

$$
f(x) \geq \min f\left[p_{n}, p_{n+1}\right] \geq \min f\left[p_{n}, n_{a}\left(p_{n}\right)\right]=f\left(p_{n+1}\right),
$$

and $f(x) \leq f\left(p_{n}\right)=a\left(p_{n}\right)$, since $x \leq p_{n+1} \leq n_{a}\left(p_{n}\right)$.

Lemma 3.7 is thus proved by induction.

Remark 3.9. Since $f(-\infty, 0]=(-\infty, \infty)$, the same argument shows that there exists a sequence $q_{1}, q_{2}, \ldots$, such that

$$
\begin{gathered}
0>q_{k}>q_{k+1} \quad \forall k \geq 1, \\
f\left(q_{k}\right) f\left(q_{k+1}\right)<0 \quad \forall k \geq 1, \\
\left|f\left(q_{k}\right)\right|<\left|f\left(q_{k+2}\right)\right| \quad \forall k \geq 1, \\
f\left(q_{k}\right)= \begin{cases}\max f\left[q_{k}, 0\right] & \text { if } f\left(q_{k}\right)>0, \\
\min f\left[q_{k}, 0\right] & \text { if } f\left(q_{k}\right)<0,\end{cases} \\
f(x) \in\left[f\left(q_{k}\right), f\left(q_{k+1}\right)\right] \quad \text { or }\left[f\left(q_{k+1}\right), f\left(q_{k}\right)\right] \quad \text { for } x \in\left[q_{k+1}, q_{k}\right] .
\end{gathered}
$$

Note that if $f\left(q_{1}\right)<0$, we can consider another sequence $q_{k}^{\prime}=q_{k+1}, k \geq 1$, and (3.64) holds for $q_{k}^{\prime}$ as well. Therefore, we may assume that $f\left(q_{1}\right)>0$. Since $\left|f\left(p_{k}\right)\right| \rightarrow \infty$, there exists $n$ such that $f\left(p_{2 n}\right)<f\left(q_{4}\right)<0$.

Let

$$
\begin{aligned}
& q=\sup \left\{x \in\left[q_{5}, q_{4}\right]: f(x)=f\left(q_{3}\right)\right\} \\
& p=\inf \left\{x \in\left[q_{3}, p_{2 n}\right]: f(x)=f\left(q_{4}\right)\right\}
\end{aligned}
$$

Note that $f\left[q, q_{4}\right]=f\left[q_{4}, q_{3}\right]=\left[f\left(q_{4}\right), f\left(q_{3}\right)\right]$.

Since $\min f\left[q_{2}, 0\right]=f\left(q_{2}\right)>f\left(q_{4}\right)$ and $f\left[q_{3}, q_{2}\right] \subset\left[f\left(q_{2}\right), f\left(q_{3}\right)\right]$, we conclude that $p$ is nonegative. Moreover, $p \neq 0$, since $f(p)=f\left(q_{4}\right) \neq 0$.

Therefore $p>0$. Note that it is possible, that $f\left[q_{3}, p\right]$ contains some points $f(x)>$ $f\left(q_{3}\right)$. If not, that is, $f\left[q_{3}, p\right]=\left[f\left(q_{4}\right), f\left(q_{3}\right)\right]$, one has $C \leq\left|\left[f\left(q_{4}\right), f\left(q_{3}\right)\right]\right| /|[q, p]| \leq$ $1 / 3$. Note that in this case the equality $C=1 / 3$ would imply that $f(x)=f\left(q_{3}\right)+q_{3}-x$ on $\left[q_{3}, p\right] \supset\left[q_{3}, 0\right]$. Then $f$ has at most one root in $\left[q_{3}, 0\right]$, which is impossible since $f$ has at least three roots in this interval: a root in $\left(q_{3}, q_{2}\right)$, a root in $\left(q_{2}, q_{1}\right)$, and a root at zero. Therefore, $C<1 / 3$. 
If, however, $f\left[q_{3}, p\right]$ contains some points $f(x)>f\left(q_{3}\right)$, then consider

$$
\begin{aligned}
& r_{1}=\inf \left\{x \in[0, p]: f(x)=f\left(q_{3}\right)\right\}, \\
& r_{2}=\inf \left\{x \in\left[q_{3}, r_{1}\right]: f(x)=\min f\left[q_{3}, r_{1}\right]\right\} .
\end{aligned}
$$

Since $r_{2}<r_{1} \leq p$, we conclude that $f\left(r_{2}\right) \geq f(p)=f\left(q_{4}\right)$. In order to finish the proof it suffices to consider

$$
r_{3}=\sup \left\{x \in\left[q_{4}, q_{3}\right]: f(x)=f\left(r_{2}\right)\right\}
$$

and to note that

$$
f\left[r_{3}, q_{3}\right]=f\left[q_{3}, r_{2}\right]=f\left[r_{2}, r_{1}\right]=\left[f\left(r_{2}\right), f\left(q_{3}\right)\right]
$$

therefore $C \leq 1 / 3$. Again, if here $C=1 / 3$, then $f$ is linear on $\left[q_{3}, r_{2}\right]$ and is linear on $\left[r_{2}, r_{1}\right]$. This means that $f$ has at most two roots in $\left[q_{3}, r_{1}\right] \supset\left[q_{3}, 0\right]$, which is not true, since $f$ has at least three roots in this interval. Therefore, in this case $C<1 / 3$ as well.

So we have finished completely the case when $M$, the set of roots of $f$, is unbounded. Case 2. $M$ is bounded.

In this case there exists $x_{0} \in M$ and a strictly monotone sequence $\left\{x_{n}\right\} \subset M$ such that $x_{n} \rightarrow x_{0}$. Without loss of generality we may assume that $x_{0}=0$ and $x_{n}>0$.

Lemma 3.10. Let $f: \mathbb{R} \rightarrow \mathbb{R}$ be 1-Lipschitz and C-BNC. If there exist $x_{n} \downarrow 0$ such that $f\left(x_{n}\right)$ $=f(0)=0$ and there exists $n_{0}$ such that $a\left(x_{n_{0}}\right)=0$ or $b\left(x_{n_{0}}\right)=0$, then $C<1 / 3$.

Proof. Assume $b\left(x_{n_{0}}\right)=0$, then there exists $n_{1}>n_{0}$ such that $x_{n_{1}}+a\left(x_{n_{1}}\right)<x_{n_{0}}$. This follows from the fact that $0 \leq a(x) \leq x$ ( $f$ is 1-Lipschitz) and $x_{n} \downarrow 0$. For such $x_{n_{1}}$, one has $b\left(x_{n_{1}}\right)=b\left(x_{n_{0}}\right)=0$. Then $f\left[0, x_{n_{1}}+a\left(x_{n_{1}}\right)\right]=f\left[0, x_{n_{1}}\right]$. Since $\left[0, x_{n_{0}}\right]$ contains more than 4 roots, Corollary 3.4 implies that $C<1 / 3$.

We will consider two cases (in analogy with (3.39), (3.40), and (3.41)):

(I) there exists $\delta>0$ such that $f$ has no roots in $(-\delta, 0)$;

(II) there exists a sequence $\left\{y_{n}\right\} \subset M, y_{n}<0$, such that $y_{n} \rightarrow 0$.

Now for nonnegative $t$ we will use the following notation:

$$
\begin{aligned}
& n_{a}^{\prime}(t)=\sup \left\{0<t^{\prime}<t: a\left(t^{\prime}\right)<a(t)\right\}, \\
& n_{b}^{\prime}(t)=\sup \left\{0<t^{\prime}<t: b\left(t^{\prime}\right)<b(t)\right\}, \\
m_{a}^{\prime}(t)= & \inf \left\{t^{\prime} \in\left[n_{b}^{\prime}(t), t\right]: f\left(t^{\prime}\right)=\max f\left[n_{b}^{\prime}(t), t\right]\right\}, \\
m_{b}^{\prime}(t)= & \inf \left\{t^{\prime} \in\left[n_{a}^{\prime}(t), t\right]: f\left(t^{\prime}\right)=\min f\left[n_{a}^{\prime}(t), t\right]\right\} .
\end{aligned}
$$

Assume (I) holds. Since $f$ has no roots in $(-\delta, 0)$, its sign is constant on this interval. Suppose, for example, that $f$ is strictly positive on $(-\delta, 0)$.

Take an interval $I=\left[0, x_{n}\right]$ for some $x_{n}<2 \delta$. In view of Lemma 3.10 we may assume $a\left(x_{n}\right)>0$ and $b\left(x_{n}\right)>0$. Since $a\left(x_{s}\right), b\left(x_{s}\right) \rightarrow 0$ there exists $x_{n+k}<x_{n}$ such that $0<a=$ $a\left(x_{n+k}\right)<a\left(x_{n}\right)$ and $0<b=b\left(x_{n+k}\right)<b\left(x_{n}\right)$. If $a \geq b$, then $f\left[-a, x_{n+k}+b\right]=f\left[0, x_{n+k}\right]$ $\left(-\delta<-x_{n} / 2<-x_{n+k} / 2<-a\right)$. By Corollary 3.4 this equality implies $C<1 / 3$. 
If $a<b$, we compare $n_{a}\left(x_{n+k}\right), n_{b}\left(x_{n+k}\right) \in\left[x_{n+k}, x_{n}\right]$ and apply the same procedure as in the proof of Lemma 3.6 (in the case when all roots of $f$ were positive and there was a sequence of them tending to infinity).

In order to prove that $C<1 / 3$ in the second case (II), we use the following lemma.

Lemma 3.11. Let $f: \mathbb{R} \rightarrow \mathbb{R}$ be 1-Lipschitz. If there exist $x_{n} \downarrow 0$ such that $f\left(x_{n}\right)=f(0)=0$, then there exists a sequence $p_{1}, p_{2}, \ldots$ of points such that

$$
\begin{gathered}
0<p_{k+1}<p_{k} \quad \forall k \geq 1, \\
f\left(p_{1}\right)>0, \quad f\left(p_{k}\right) f\left(p_{k+1}\right)<0 \quad \forall k \geq 1, \\
\left|f\left(p_{k}\right)\right|>\left|f\left(p_{k+2}\right)\right| \quad \forall k \geq 1, \\
f\left(p_{k}\right)=a\left(p_{k}\right) \quad \text { if } f\left(p_{k}\right)>0, \quad f\left(p_{k}\right)=-b\left(p_{k}\right) \quad \text { if } f\left(p_{k}\right)<0, \\
f(x) \in\left[f\left(p_{k}\right), f\left(p_{k+1}\right)\right] \quad \text { or }\left[f\left(p_{k+1}\right), f\left(p_{k}\right)\right] \quad \text { for } x \in\left[p_{k+1}, p_{k}\right] .
\end{gathered}
$$

Remark 3.12. Since $a(x), b(x) \rightarrow 0$ as $x \downarrow 0$, the fourth condition implies $\left|f\left(p_{k}\right)\right| \rightarrow 0$.

Proof. Let $z_{1}=\inf \left\{z \in\left[0, x_{1}\right]: f(z)=a\left(x_{1}\right)\right\}$ and $z_{2}=\inf \left\{z \in\left[0, x_{1}\right]: f(z)=-b\left(x_{1}\right)\right\}$. Without loss of generality assume that $z_{1}>z_{2}$. Then put $p_{1}=z_{1}$ and $p_{2}=m_{b}^{\prime}\left(z_{1}\right)$.

Having constructed $p_{1}>\cdots>p_{n-1}>p_{n}>0$, we put

$$
p_{n+1}= \begin{cases}m_{b}^{\prime}\left(p_{n}\right) & \text { if } f\left(p_{n}\right)>0, \\ m_{a}^{\prime}\left(p_{n}\right) & \text { if } f\left(p_{n}\right)<0\end{cases}
$$

Since $a(x), b(x) \rightarrow 0$ as $x \rightarrow 0$ and $a(x), b(x)>0$ for all $x$ (we may assume this, because otherwise Lemma 3.10 immediately implies $C<1 / 3$ ), we conclude that $p_{n+1}>0$.

All the conditions are verified in the same way as in Lemma 3.7.

Remark 3.13. As before, note that we can construct a sequence $q_{1}<q_{2}<\cdots<0$ with analogous properties. We may assume that $f\left(q_{1}\right)<0$.

Since $f\left(p_{n}\right) \rightarrow 0$, there exists $k \geq 2$ such that $0>f\left(p_{2 k-2}\right)>f\left(q_{1}\right)$. Let

$$
\begin{gathered}
q=\sup \left\{x \in\left[q_{1}, p_{2 k-1}\right]: f(x)=f\left(p_{2 k-2}\right)\right\}, \\
p=\inf \left\{x \in\left[p_{2 k-2}, p_{2 k-3}\right]: f(x)=f\left(p_{2 k-1}\right)\right\} .
\end{gathered}
$$

Note that $f\left[p_{2 k-1}, p_{2 k-2}\right]=f\left[p_{2 k-2}, p\right]=\left[f\left(p_{2 k-2}\right), f\left(p_{2 k-1}\right)\right]$.

Note also that $q \leq 0$, since $f$ does not attain the value $f\left(p_{2 k-2}\right)$ on $\left[0, p_{2 k-1}\right]$. Also, $q$ cannot be zero, since $f(q)=f\left(p_{2 k-2}\right) \neq 0$.

So $q<0$. It is possible that $f\left[q, p_{2 k-1}\right]$ contains some points $f(x)>f\left(p_{2 k-1}\right)$. If not, that is, $f\left[q, p_{2 k-1}\right]=\left[f\left(p_{2 k-2}\right), f\left(p_{2 k-1}\right)\right]$, one has $C \leq 1 / 3$. Note that in this case the equality $C=1 / 3$ would imply that $f(x)$ is linear on $\left[q, p_{2 k-1}\right]$ which is impossible since $f$ has infinitely many roots in this interval. Therefore, $C<1 / 3$.

If, however, $f\left[q, p_{2 k-1}\right]$ contains some points $f(x)>f\left(p_{2 k-1}\right)$, then consider

$$
\begin{gathered}
r_{1}=\sup \left\{x \in[q, 0]: f(x)=f\left(p_{2 k-1}\right)\right\}, \\
r_{2}=\sup \left\{x \in\left[r_{1}, p_{2 k-1}\right]: f(x)=\min f\left[r_{1}, p_{2 k-1}\right]\right\} .
\end{gathered}
$$


Since $r_{2}>r_{1} \geq q$, we conclude that $f\left(r_{2}\right) \geq f(q)=f\left(p_{2 k-2}\right)$. To finish the proof, consider

$$
r_{3}=\inf \left\{x \in\left[p_{2 k-1}, p_{2 k-2}\right]: f(x)=f\left(r_{2}\right)\right\}
$$

and note that

$$
f\left[r_{1}, r_{2}\right]=f\left[r_{2}, p_{2 k-1}\right]=f\left[p_{2 k-1}, r_{3}\right]=\left[f\left(r_{2}\right), f\left(p_{2 k-1}\right)\right],
$$

and therefore $C \leq 1 / 3$. Again, if here $C=1 / 3$, then $f$ is linear on $\left[r_{1}, r_{2}\right]$ and is linear on $\left[r_{2}, p_{2 k-1}\right]$. This means that $f$ has at most two roots in $\left[r_{1}, 0\right]$, which is not true, since $f$ has infinitely many roots in this interval. Therefore, also in this case $C<1 / 3$.

This finishes the treatment of the case when $M$ is bounded, as well as the proof of the theorem.

\section{Acknowledgment}

I would like to thank Professor Gideon Schechtman for stimulating discussions and the organizers of the Small Sets in Analysis Workshop which took place at Technion, Haifa, Israel, for their support and hospitality.

\section{References}

[1] H. Federer, Geometric Measure Theory, Springer, Berlin, 1996.

[2] G. David and S. Semmes, Regular mappings between dimensions, Publ. Mat. 44 (2000), no. 2, 369-417.

[3] W. B. Johnson, J. Lindenstrauss, D. Preiss, and G. Schechtman, Uniform quotient mappings of the plane, Michigan Math. J. 47 (2000), no. 1, 15-31.

[4] O. Maleva, Lipschitz quotient mappings with good ratio of constants, Mathematika 49 (2002), no. 1-2, p. 159-165.

[5] _ Point preimages under ball non-collapsing mappings, Geometric Aspects of Functional Analysis, Lecture Notes in Math., vol. 1807, Springer, Berlin, 2003, pp. 148-157.

[6] Components of level sets of uniform co-Lipschitz functions on the plane, Proc. Amer. Math. Soc. 133 (2005), no. 3, 841-850.

[7] S. Rickman, Quasiregular Mappings, Ergebnisse der Mathematik und ihrer Grenzgebiete (3), vol. 26, Springer, Berlin, 1993.

Olga Maleva: Department of Mathematics, Weizmann Institute of Science, Rehovot 76100, Israel

Current address: Department of Mathematics, University College London, Gower Street, London WC1E 6BT, United Kingdom

E-mail address: olga@math.ucl.ac.uk 


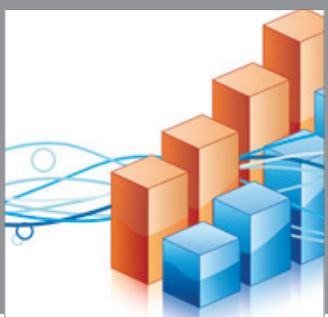

Advances in

Operations Research

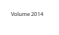

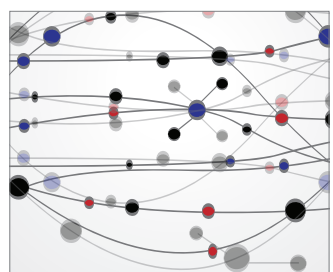

\section{The Scientific} World Journal
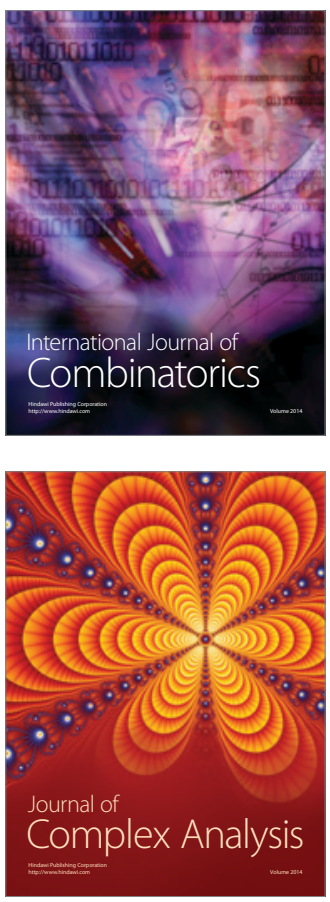

International Journal of

Mathematics and

Mathematical

Sciences
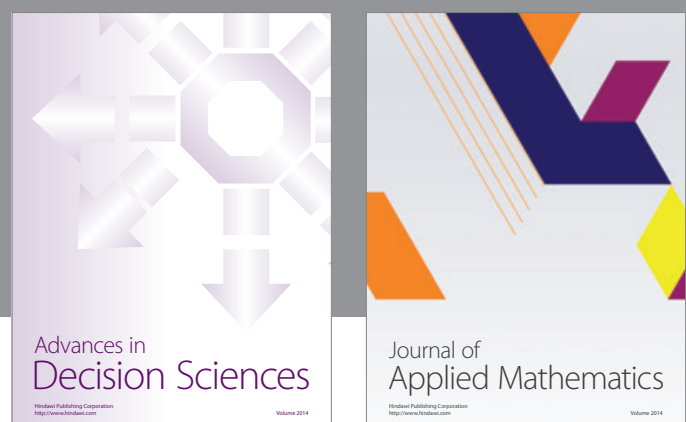

Journal of

Applied Mathematics
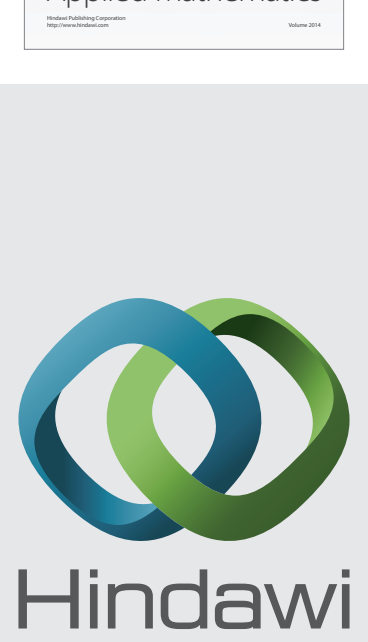

Submit your manuscripts at http://www.hindawi.com
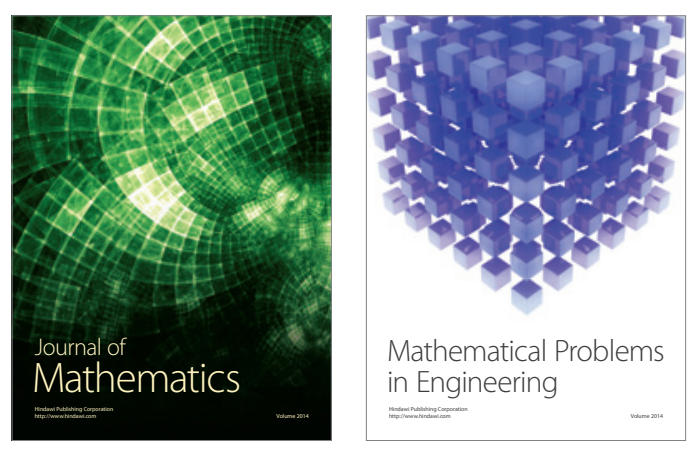

Mathematical Problems in Engineering
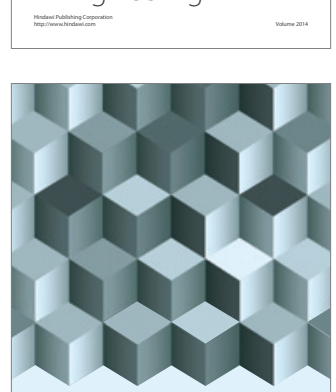

Journal of

Function Spaces
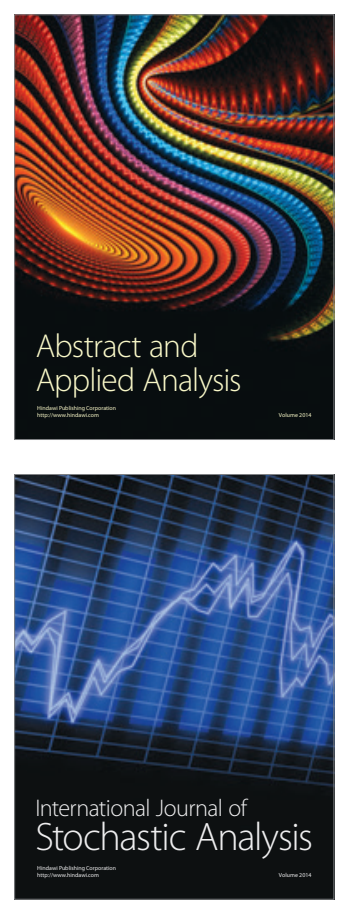

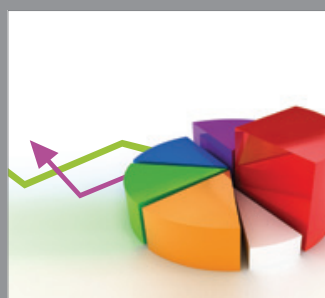

ournal of

Probability and Statistics

Promensencen
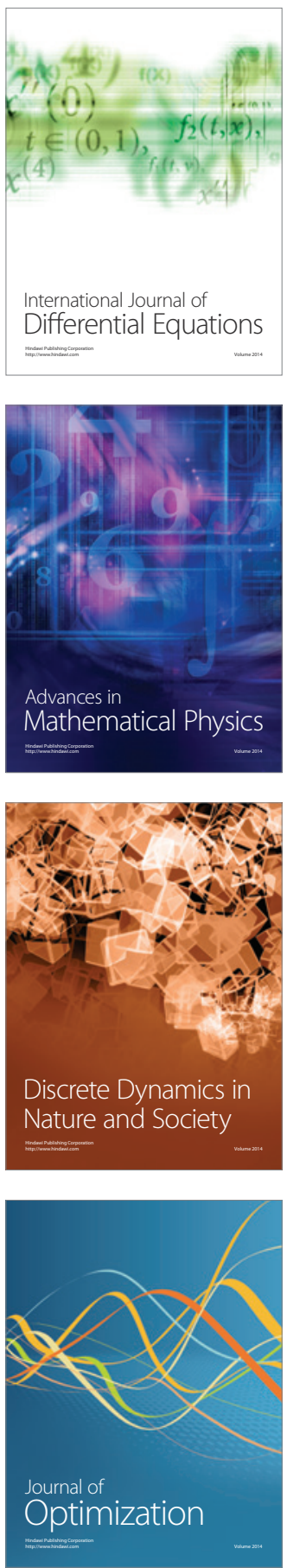